\title{
Laser Doppler velocimeter for laboratory training
}

Ivan Fedosov, Boris Djagilev, Alexey Kurtov, Valery Tuchin

Ivan V. Fedosov, Boris L. Djagilev, Alexey S. Kurtov, Valery V. Tuchin, "Laser Doppler velocimeter for laboratory training," Proc. SPIE 4588, Seventh International Conference on Education and Training in Optics and Photonics, (28 May 2002); doi: 10.1117/12.468743

SPIE Event: Education and Training in Optics and Photonics 2001, 2001, Singapore, Singapore 


\title{
Laser Doppler velocimeter for laboratory training
}

\author{
Ivan V. Fedosov ${ }^{*}$, Boris L. Djagilev, Alexey S. Kurtov, Valery V. Tuchin \\ Saratov State University, REC-006, Laboratory of biophotonics.
}

\begin{abstract}
The laser Doppler velocimeter was developed specially for students laboratory training. The experimental kit consists of the classic dual beam laser Doppler system and the set of the dynamic objects such as moving phase screen and scattering flows. Signal processing and data analysis is performed using personal computer that allows for flexible training.
\end{abstract}

Keywords: speckles, blood flow

\section{INTRODUCTION}

Optical education in the century of hi-tech instrumentation has to reveal the interrelations between optics and different branches of sciences and the principles of optical instrumentation design for students. Practical training is the shortest way to demonstrate to students the interrelations between the fundamental principles and its real world applications. At present laser Doppler anemometry is one of well developed and useful instrument with wide range of applications ${ }^{1}$. Laser Doppler velocimeters (LDV) are used for supersonic flow investigation, for multicomponent flow analysis ${ }^{2}$, in vivo bioflow monitoring ${ }^{3}$ etc. LDV is based on the fundamental principles of the modern optics such coherence, Doppler effect, light scattering and diffraction. In one hand, practical work with the simplest LDV will illustrate these principles, will enable students to understand the principles of optical instrumentation design. In other hand such simple device is a useful instrument for practical investigation of flow structure that will allow for students to arrange its research works in mechanics and biophysics. LDV is one of examples of interrelations between different branches of modern physics. Moreover the flexible signal processing using personal computer illustrates the principles of digital signal processing and analysis.

Our main idea is to develop the basic kit of instruments to provide the wide range of practical works on optics, fluid mechanics, biophysics, engineering and digital signal processing. In present paper the ideology of such kit is described and illustrated with the first result obtained with our first prototype. The examples of practical tasks are proposed and discussed.

\section{BASIC KIT}

This practical work will enable student to: understand principles of optical heterodyning; understand principles of digital signal processing; get an overview of principles and schemes for basic laser instruments such LDV. Basic kit consists on the simple dual beam LDV designed for forward scattering geometry; positioning stage with micrometer screw; frozen glass rotating disc with motor drive; set of transparent tubes and scattering suspension. The main component of basic kit is the LDV setup that is shown on fig. 1. Coherent collimated light beam from $1 \mathrm{~mW}$ laser diode module is divided with wavefront splitter and resulting beams are focused into the tube or on the glass disc surface and forms the sensitive volume. Angle between crossed beams is $10 \mathrm{deg}$. Scattered light is collected on the photodiode and non-scattered light is blocked with beam stops. Objectives working distances are $67 \mathrm{~mm}$. The optical components are mounted on the steel plate. Photocurrent signal is amplified and digitized with standard PC sound card. Photocurrent amplifier output is designed for sound card to minimize the cost of educational set up. The further signal processing is performed using standard or customized software and allows for different kind of work. The additional equipment is mounted on the micropositioning stage. Motorized frozen glass disc of $14 \mathrm{~mm}$ in diameter is driven with variable DC voltage $0-24$ volts. The results of spectrum analysis of the photocurrent signal obtained when frozen glass disc is placed in the sensitive volume of the LDV is shown on the figure 2 .

\footnotetext{
${ }^{*}$ FedosovIV@info.sgu.ru; phone: 7(8452)51-51-95; Saratov State University', Dept. of Optics, 155, Moskovskaya, 410026 Saratov. Russia.
} 


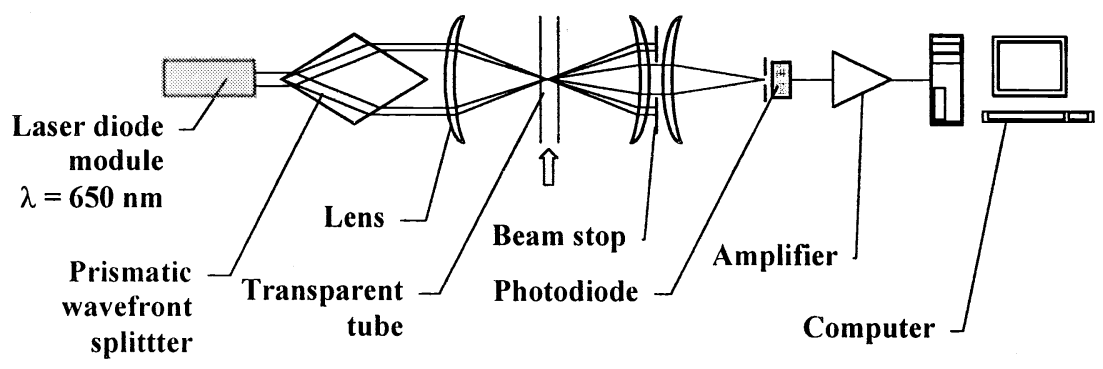

Fig. 1. Laser Doppler velocimeter

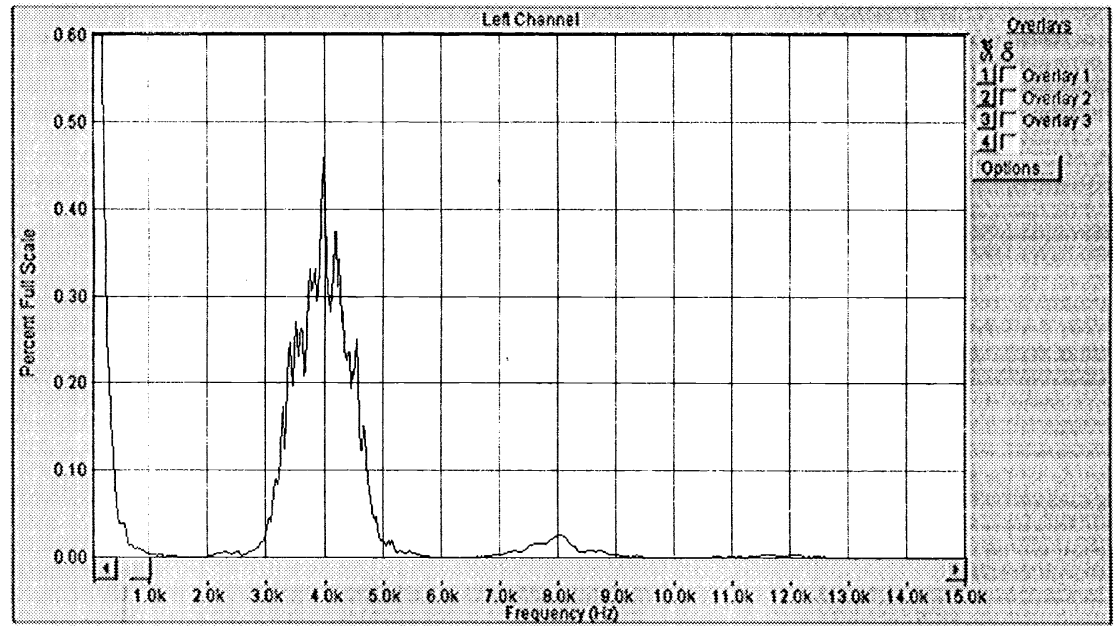

Fig. 2. Doppler frequency shift power spectrum obtained with rotating frozen glass disk.

\section{EXAMPLES OF TASKS FOR STUDENTS}

The developed kit allows for different kinds of tasks for students.

The simplest work is the investigation of the LDV properties. This work includes the following tasks: to calculate the relation of the object velocity and Doppler frequency shift at known scattering geometry; to measure velocity of frozen glass disc surface for different voltage on disc driver using two methods: LDV and direct measurements of rotation period and disc radius for different; to analyze the correlation between two resulting data sets. The additional task is to measure the length of LDV sensitive volume using rotating disk placed on the micropositionning stage or to investigate the case when the disc surface is not perpendicular to the optical axe of the instrument and to check the property of to measure velocity projection on the vector of sensitivity ${ }^{4}$.

Another kind of work is the investigation of flow and bioflow dynamics. Such research includes the following tasks: to determine the velocity distribution across the flow in tube; to investigate the flow velocity distribution for liquids with different viscosity at fixed pressure difference on the edges of tube. The additional task for students of biomedical specialization is the investigation of whole blood flow as the flow of non-Newtonian liquid.

The third group of practical tasks is the investigation of digital signal processing principles and development of softwarc for the computer assisted experimental setup. LDV is used as a source of real signal. Typical tasks of this group are: the digital filtering algorithm comparison; digital spectrum analysis of stochastic signal; automatic velocity monitoring. 


\section{CONCLUSION}

The universal LDV for practical works is developed to support different kinds of courses on optics; optical instrumentation and measurements; fluid mechanics; digital signal processing. Moreover such device is the useful laboratory instrument for student's scientific research support. The basic kit is designed for use with standard PC with minimal system requirement and contains no one costly component.

\section{ACKNOWLEGEMENTS}

This work is supported U. S. Civilian Research \& Development Foundation for the Independent States of the Former Soviet Union, Award N REC-006.

\section{REFERENCES}

1. C. Tropea, "Laser Doppler anemometry: recent develoments and future challenges," Meas Sci. Technol. 6, pp. 605-619, 1995.

2. F. Durst, G. Brenn, T.H. Xu, "A review of the development and characteristics of planar phase - Doppler anemometry," Meas. Schi. Technol., 8, pp. 1203-1221, 1997.

3. T. Eiju, M. Nagai, K. Matsuda, J. Ohtsubo, K. Homma, K. Shimizu, "Microscopic laser Doppler Velocimeter for blood velocity measurement," Opt. Eng., Vol. 32, No. 1, pp. 15-20.

4. B. S. Rinkevitchus, Laser diagnostics of flows, Moscow Institute of Energetic Engineering Press, Moskow, 1990. 